\title{
Beam quality study for a grating-based dielectric laser-driven accelerator
}

Y. Wei, S. Jamison, G. Xia, K. Hanahoe, Y. Li, J. D. A. Smith, and C. P. Welsch

Citation: Physics of Plasmas 24, 023102 (2017); doi: 10.1063/1.4975080

View online: https://doi.org/10.1063/1.4975080

View Table of Contents: http://aip.scitation.org/toc/php/24/2

Published by the American Institute of Physics

\section{Articles you may be interested in}

General theory of the plasmoid instability

Physics of Plasmas 23, 100702 (2016); 10.1063/1.4964481

Dual-gratings with a Bragg reflector for dielectric laser-driven accelerators

Physics of Plasmas 24, 073115 (2017); 10.1063/1.4993206

Electron holes in phase space: What they are and why they matter

Physics of Plasmas 24, 055601 (2017); 10.1063/1.4976854

Particle acceleration in laser-driven magnetic reconnection

Physics of Plasmas 24, 041408 (2017); 10.1063/1.4978627

Breaking of dynamical adiabaticity in direct laser acceleration of electrons

Physics of Plasmas 24, 023101 (2017); 10.1063/1.4975076

Particle dynamics in the electron current layer in collisionless magnetic reconnection

Physics of Plasmas 23, 102102 (2016); 10.1063/1.4963008 


\title{
Beam quality study for a grating-based dielectric laser-driven accelerator
}

\author{
Y. Wei, ${ }^{1,2, a)}$ S. Jamison, ${ }^{3}$ G. Xia,,${ }^{1,4}$ K. Hanahoe, ${ }^{1,4}$ Y. Li, ${ }^{1,4}$ J. D. A. Smith, ${ }^{5}$ \\ and C. P. Welsch ${ }^{1,2}$ \\ ${ }^{1}$ Cockcroft Institute, Sci-Tech Daresbury, Warrington WA4 4AD, United Kingdom \\ ${ }^{2}$ Physics Department, University of Liverpool, Liverpool L69 3BX, United Kingdom \\ ${ }^{3}$ Accelerator Science and Technology Centre, Sci-Tech Daresbury, Warrington WA4 4AD, United Kingdom \\ ${ }^{4}$ School of Physics and Astronomy, University of Manchester, Manchester M13 9PL, United Kingdom \\ ${ }^{5}$ Tech-X UK Ltd, Sci-Tech Daresbury, Warrington WA4 4AD, United Kingdom
}

(Received 12 September 2016; accepted 13 January 2017; published online 1 February 2017)

Dielectric laser-driven accelerators (DLAs) based on grating structures are considered to be one of the most promising technologies to reduce the size and cost of future particle accelerators. They offer high accelerating gradients of up to several $\mathrm{GV} / \mathrm{m}$ in combination with mature lithographic techniques for structure fabrication. This paper numerically investigates the beam quality for acceleration of electrons in a realistic dual-grating DLA. In our simulations, we use beam parameters of the future Compact Linear Accelerator for Research and Applications facility to load an electron bunch into an optimized 100-period dual-grating structure where it interacts with a realistic laser pulse. The emittance, energy spread, and loaded accelerating gradient for modulated electrons are then analyzed in detail. Results from simulations show that an accelerating gradient of up to $1.13 \pm 0.15 \mathrm{GV} / \mathrm{m}$ with an extremely small emittance growth, $3.6 \%$, can be expected. Published by AIP Publishing. [http://dx.doi.org/10.1063/1.4975080]

\section{INTRODUCTION}

Accelerating gradients for conventional RF-cavity-based particle accelerators are usually in the range of $20-50 \mathrm{MV} / \mathrm{m}$ and are severely limited by RF-induced metal surface breakdown. ${ }^{1-3}$ Dielectric materials can withstand electric fields roughly 2 orders of magnitude larger than metals at optical frequencies, and together with large electric fields from ultra-short laser pulses, they enable a new acceleration scheme of dielectric laser-driven accelerators (DLAs) that support accelerating gradients up to several $\mathrm{GV} / \mathrm{m}$. Many candidates for DLAs have been proposed: grating-based structures, ${ }^{4-7}$ photonic crystal structures, ${ }^{8-10}$ and woodpile structures. ${ }^{11}$ Dual-grating structures are of particular interest because they have simpler structural geometry compared to other types of DLAs. In addition, they can also be used as undulators, ${ }^{12}$ beam position monitors, ${ }^{13}$ and deflecting and focusing structures. ${ }^{14}$ These structures can be integrated on a single wafer by using existing nanofabrication technology with nanometer precision and low cost. So far, two experiments have successfully demonstrated high accelerating gradients of $300 \mathrm{MV} / \mathrm{m}^{15}$ and $690 \mathrm{MV} / \mathrm{m}^{16}$ for relativistic electron acceleration in fused silica dual-grating structures. As for non-relativistic electron acceleration, accelerating gradients of $25 \mathrm{MV} / \mathrm{m}^{17}$ for fused silica, and $220 \mathrm{MV} / \mathrm{m}^{18}$ and $370 \mathrm{MV} / \mathrm{m}^{19}$ for silicon structures have previously been observed.

Optimal geometry studies of dual-grating structures have already been performed with the aim to increase the maximum accelerating gradient. ${ }^{4-6,20}$ However, few studies have been conducted into the particle beam quality ${ }^{21}$ that can be obtained from a DLA, despite it being one of the

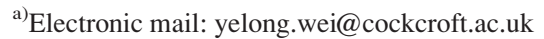

most essential parameters for any accelerator. In this paper, we report on geometry optimization and beam quality studies for dual-grating DLAs. In Section II, we present theoretical descriptions for the electromagnetic field in a dual-grating structure and geometry optimization to maximize the accelerating factor with the widest channel gap. This is followed by a detailed beam quality study for an optimized 100-period dual-grating structure in terms of emittance, energy spread, and loaded accelerating gradient in Section III. Simulations were performed using beam properties of the future Compact Linear Accelerator for Research and Applications (CLARA), ${ }^{22}$ which is a planned $\mathrm{x}$-ray free electron laser (FEL) test facility to be located at the Daresbury laboratory, UK. Finally, the limitations on the electron energy gain in our simulations are also discussed.

\section{THEORY AND GEOMETRY OPTIMIZATION}

Evanescent electromagnetic fields close to a single grating structure excited by a plane wave have been explored by Palmer, ${ }^{23}$ and Pai and Awada. ${ }^{24}$ Based on the same method, we can describe the electric and magnetic fields for a dualgrating structure, which is illuminated by a single laser beam as shown in Fig. 1. For a linear polarized TM mode, the transverse magnetic field $B_{\mathrm{x}}$ for the $n$th $(n=1,2,3, \ldots)$ order grating spatial mode can be given by ${ }^{25}$

$$
\begin{aligned}
B_{\mathrm{x}, \mathrm{n}}= & \left(D_{1, \mathrm{n}} e^{-k_{\mathrm{y}} y}+D_{2, \mathrm{n}} e^{+k_{\mathrm{y}} y}\right) e^{i n k_{\mathrm{z}} z-i \omega_{0} t+i \varphi} \\
= & \left(\left(D_{1, \mathrm{n}}+D_{2, \mathrm{n}}\right) \cosh \left(k_{\mathrm{y}} y\right)\right. \\
& \left.+\left(D_{2, \mathrm{n}}-D_{1, \mathrm{n}}\right) \sinh \left(k_{\mathrm{y}} y\right)\right) e^{i n k_{z} z-i \omega_{0} t+i \varphi},
\end{aligned}
$$

where $D_{1, \mathrm{n}}$ and $D_{2, \mathrm{n}}$ are the coefficients of the transmitted and reflected propagating modes, $k_{\mathrm{y}}$ is the transverse wave number, $k_{\mathrm{y}}=2 \pi / \beta \gamma \lambda_{0}, \beta=v / c$ for electron velocity, 


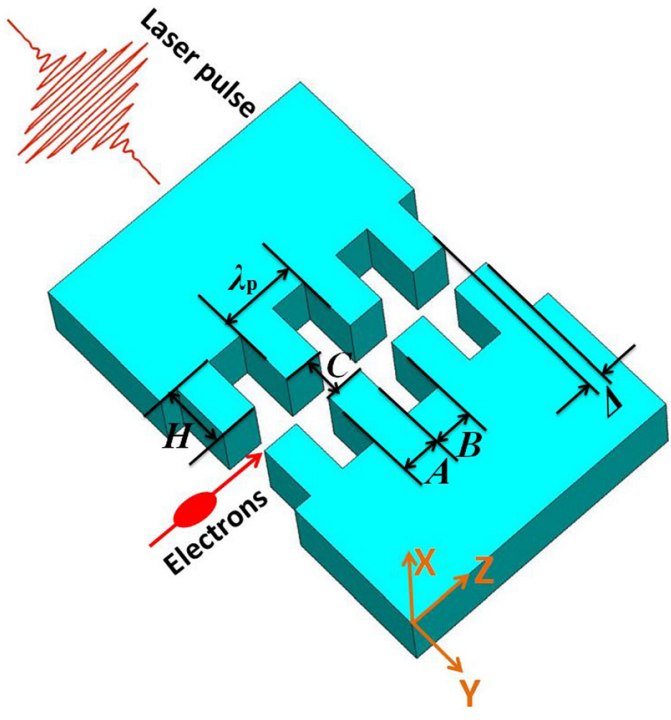

FIG. 1. Schematic of a dual-grating structure. $\lambda_{\mathrm{p}}, A, B, C, \mathrm{H}$, and $\Delta$ represent grating period, dielectric pillar width, pillar trench, vacuum channel gap, pillar height, and longitudinal misalignment level, respectively. $A+B=\lambda_{\mathrm{p}}$ is selected for all simulations.

$\gamma=\left(1-\beta^{2}\right)^{-0.5}, \omega_{0}$ and $\lambda_{0}$ are laser angular frequency and wavelength, respectively, and $k_{\mathrm{z}}$ is the longitudinal wave number, $k_{\mathrm{z}}=2 \pi / \beta \lambda_{0}$. For our grating structures, the fundamental mode, $n=1$, is chosen for acceleration as it has maximum acceleration gradient compared to other higher order modes. According to Maxwell's equations $\nabla \times \boldsymbol{B}=-\mu \epsilon i \omega \boldsymbol{E}$, we can easily find the expressions for $E_{\mathrm{z}}$ and $E_{\mathrm{y}}$, so the calculated Lorentz force from $\boldsymbol{F}=e(\boldsymbol{E}+v \times \boldsymbol{B})$ is described by

$$
\begin{aligned}
\boldsymbol{F} & =\frac{e c}{\beta \gamma}\left[\begin{array}{c}
0 \\
\frac{-1}{\gamma}\left(D_{\mathrm{s}} \cosh \left(k_{y} y\right)+D_{\mathrm{c}} \sinh \left(k_{y} y\right)\right) \cos \left(k_{z} z-\omega_{0} t+\varphi\right) \\
\left(D_{\mathrm{s}} \sinh \left(k_{y} y\right)+D_{\mathrm{c}} \cosh \left(k_{y} y\right)\right) \sin \left(k_{z} z-\omega_{0} t+\varphi\right)
\end{array}\right] \\
& =\left[\begin{array}{c}
0 \\
F_{y} \\
F_{z}
\end{array}\right]
\end{aligned}
$$

where $D_{\mathrm{s}}=D_{1, \mathrm{n}}+D_{2, \mathrm{n}}$ and $D_{\mathrm{c}}=D_{2, \mathrm{n}}-D_{1, \mathrm{n}}, e$ is the charge of a single electron, and $c$ is the speed of light. When $D_{\mathrm{s}}=0$ and $D_{\mathrm{c}} \neq 0$, a uniform accelerating mode as shown in Fig. 2 with a vanishing deflecting force on axis will exist in the channel, which is our desired case for the following optimization. When $D_{\mathrm{s}} \neq 0$ and $D_{\mathrm{c}}=0$, the accelerating force vanishes on axis, which is not suitable for the acceleration of electrons. For both cases, the resulting average accelerating gradient $G_{0}$ and deflecting gradient $D_{0}$ are given as follows:

$$
\begin{aligned}
& G_{0}=\frac{1}{e \lambda_{\mathrm{p}}} \int_{0}^{\lambda_{\mathrm{p}}} F_{\mathrm{z}}[z(t), t] d z, \\
& D_{0}=\frac{1}{e \lambda_{\mathrm{p}}} \int_{0}^{\lambda_{\mathrm{p}}} F_{\mathrm{y}}[z(t), t] d z .
\end{aligned}
$$

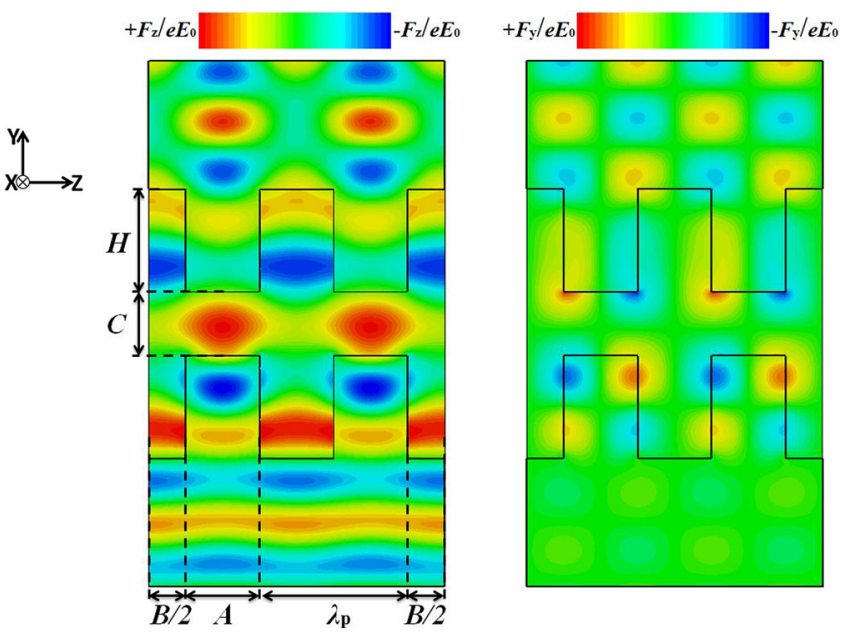

FIG. 2. Longitudinal accelerating force $F_{\mathrm{z}}$ and a weak deflecting force $F_{\mathrm{y}}$ in a dual-grating structure illuminated by an input laser field $E_{0}$ along the $\mathrm{y}$-axis.

When a plane wave with an input field of $E_{0}$ travels through a dual-grating structure, the maximum electric field $E_{\mathrm{m}}$ in the structure should not exceed the material damage field. The accelerating factor ${ }^{26} A F$ and the deflecting factor ${ }^{26} D F$ are defined as the ratios of the accelerating gradient $G_{0}$ and deflecting gradient $G_{0}$ to the maximum electric field $E_{\mathrm{m}}$, respectively, in the structure

$$
\begin{aligned}
& A F=G_{0} / E_{\mathrm{m}}, \\
& D F=D_{0} / E_{\mathrm{m}} .
\end{aligned}
$$

Our following geometry optimization aims to maximize the accelerating factor $A F$ with the widest channel gap $C$ while minimizing the deflecting factor.

In order to optimize a dual-grating structure, the highperformance particle-in-cell (PIC) code VSim, ${ }^{27}$ based on the finite difference time domain (FDTD) method, is used to compute the electric and magnetic fields generated in the structure. A uniform plane wave with a wavelength of $\lambda_{0}=2 \mu \mathrm{m}$ illuminates the bottom side of the two-period dual-grating structure, as shown in Fig. 2. A grating period of $\lambda_{\mathrm{p}}=2 \mu \mathrm{m}$ is chosen so that the phase synchronicity ${ }^{28}$ can be achieved between the first spatial harmonics $(n=1)$ and relativistic electrons $(\beta=1)$. Quartz, ${ }^{29}$ with a refractive index $n_{\mathrm{r}}=1.5$, is chosen as the grating material due to its high level of transparency in the optics frequency range, high field damage threshold, and high thermal conductivity.

The first design criterion is to generate a periodic $\pi$ phase shift for wave front in the channel center to enable synchronous with relativistic electrons. This can be achieved by initially setting the pillar height as $H=\frac{\lambda_{\mathrm{p}}}{2\left(n_{\mathrm{r}}-1\right)}=\lambda_{\mathrm{p}}$. It can be seen in Fig. 3(a) that the accelerating factor gradually decreases when the vacuum channel gap $C$ increases. Figure 3(b) shows that the deflecting factor drops with $C$ for $C \leq 0.7 \lambda_{\mathrm{p}}$, but it turns to increase when $C \geq 0.7 \lambda_{\mathrm{p}}$. A channel gap of $C=0.50 \lambda_{\mathrm{p}}$ is chosen as an acceptable parameter due to a trade-off between the accelerating gradient with a weak deflecting force and the available phase space in which high 

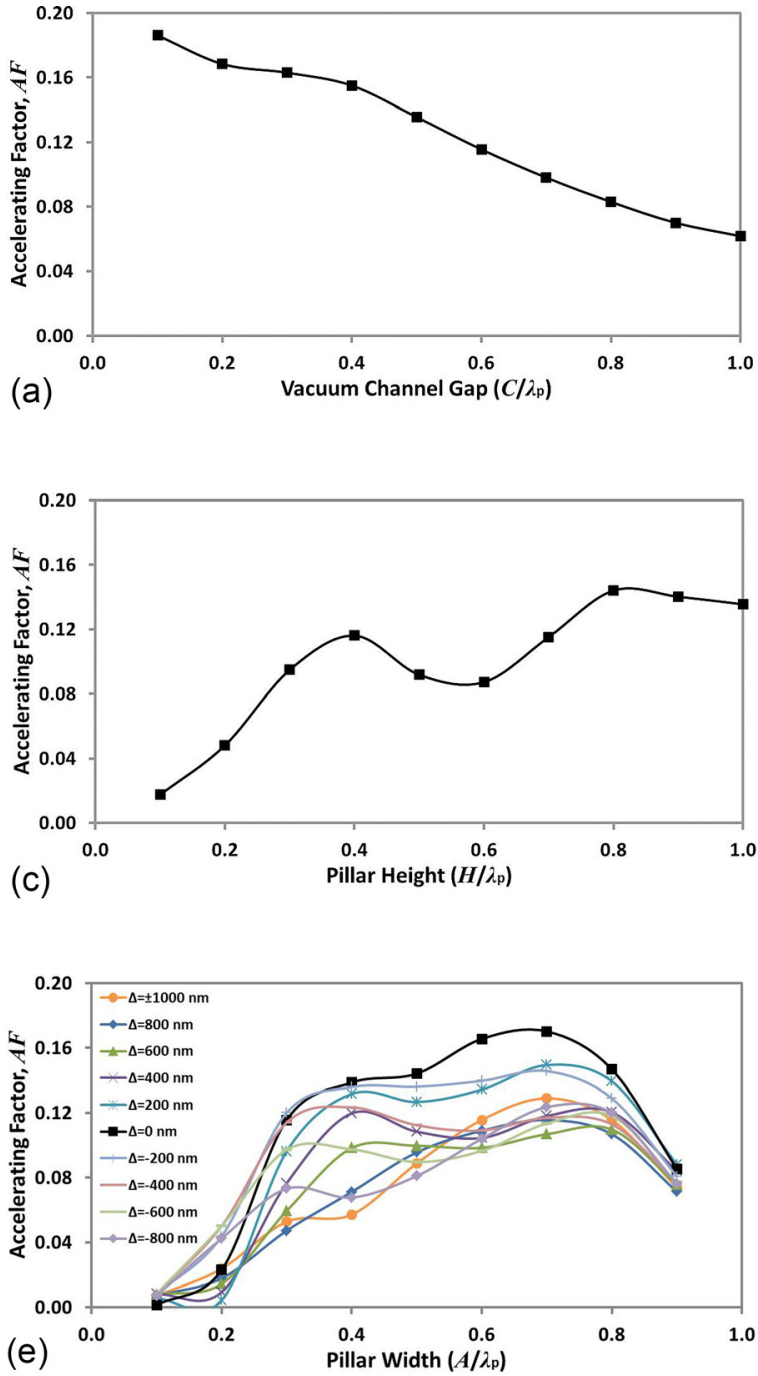
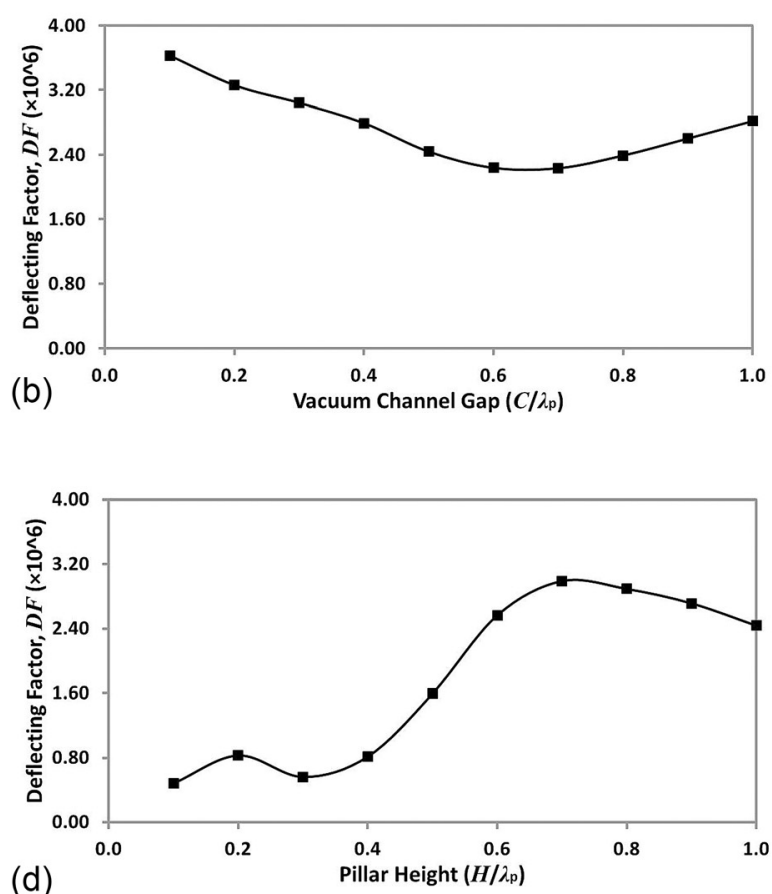

(d)

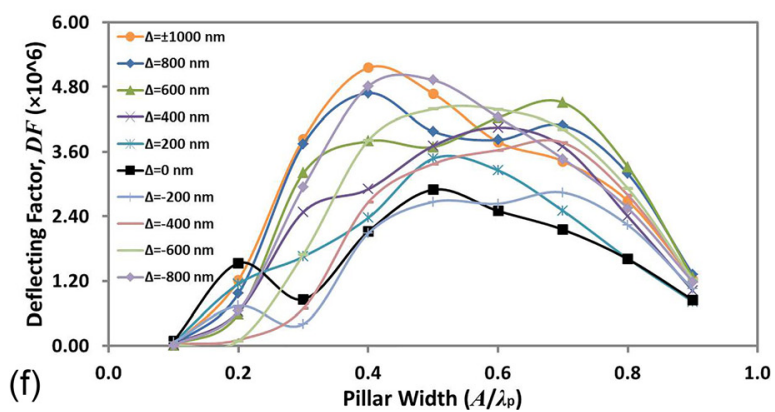

FIG. 3. Optimization for a single-period dual-grating structure, a calculated accelerating factor $A F$ ((a), (c), and (e)), and a deflecting factor $D F$ ((b), (d), and (f)) as functions of a vacuum channel gap $C\left(H=\lambda_{\mathrm{p}}, A=0.50 \lambda_{\mathrm{p}}\right.$, and $\left.\Delta=0 \mathrm{~nm}\right)$, a pillar height $H\left(C=0.50 \lambda_{\mathrm{p}}, A=0.50 \lambda_{\mathrm{p}}\right.$, and $\left.\Delta=0 \mathrm{~nm}\right)$, and a pillar width $A$ with a variable longitudinal misalignment level $\Delta\left(C=0.50 \lambda_{\mathrm{p}}, H=0.80 \lambda_{\mathrm{p}}\right)$.

acceleration occurs. Figure 3(c) shows that the maximum $A F=0.144$ can be obtained at $H=0.80 \lambda_{\mathrm{p}}$ for the structures with a fixed $C=0.50 \lambda_{\mathrm{p}}$, while the peak $D F$ occurs at $H=0.70 \lambda_{\mathrm{p}}$ as shown in Fig. 3(d). Fixing the grating, $C=0.50 \lambda_{\mathrm{p}}$ and $H=0.80 \lambda_{\mathrm{p}}$, we then set out to find the optimal pillar width $A$ and the longitudinal misalignment level $\Delta$. The simulations sweep the pillar width $A$ from $0.10 \lambda_{\mathrm{p}}$ to $0.90 \lambda_{\mathrm{p}}$ combined with the variable misalignment $\Delta$ from $-0.50 \lambda_{\mathrm{p}}$ to $0.50 \lambda_{\mathrm{p}}$ to obtain the optimum geometries. As shown in Fig. 3(e), a global maximum accelerating factor $(A F=0.170)$ can be easily found for a structure with $C=0.50 \lambda_{\mathrm{p}}, A=0.70 \lambda_{\mathrm{p}}, H=0.80 \lambda_{\mathrm{p}}$, and $\Delta=0 \mathrm{~nm}$. It is interesting to note that the structures usually perform best when perfectly aligned $(\Delta=0 \mathrm{~nm})$, which can be seen in Figs. 3(e) and 3(f). This agrees well with the results from England et al. ${ }^{30}$ where they found that the weakest transverse deflecting force appeared for gratings with the perfect alignment. The damage threshold for quartz is about $1.5 \mathrm{~J} / \mathrm{cm}^{2}$ for laser pulses of $100 \mathrm{fs},{ }^{31,32}$ which is equivalent to an electric field of $E_{\mathrm{th}}=9.0 \mathrm{GV} / \mathrm{m}$, so the maximum achievable gradient for a dual-grating structure is $0.170 \times 9.0=1.53 \mathrm{GV} / \mathrm{m}$.

\section{BEAM QUALITY STUDY}

\section{A. Optimum structure geometry}

From optimizations in Section II, a dual-grating structure with $C=0.50 \lambda_{\mathrm{p}}, H=0.80 \lambda_{\mathrm{p}}$, and $A=0.70 \lambda_{\mathrm{p}}$ was found to be desirable as an optimum choice. Such optimized dualgrating structure with 100 periods as illustrated in Fig. 5 is chosen for the following beam quality studies, and the geometry parameters are summarized in Table I.

\section{B. Laser parameters}

In the simulation, a linearly polarized Gaussian laser plane wave, as shown in Fig. 4, is launched to propagate along the $y$-axis

$$
E_{\mathrm{z}}=E_{\mathrm{p}} e^{-\left(\frac{z}{w_{\mathrm{z}}}\right)^{2}-2 \ln 2\left(\frac{t}{\tau}\right)^{2}} \cos (2 \pi f t)
$$

where $E_{\mathrm{p}}, w_{\mathrm{z}}, \tau$, and $f$ represent the peak field, $\mathrm{z}$-axis waist radius, full-width at half-maximum (FWHM) duration, and frequency, respectively. All relevant parameters are described in Table II. The peak laser field $E_{\mathrm{p}}$ is set to $5.0 \mathrm{GV} / \mathrm{m}$, and 
TABLE I. Geometry details of a 100-period dual-grating structure.

\begin{tabular}{lc}
\hline \hline Geometry \\
\hline Number of periods \\
Grating period $\lambda_{\mathrm{p}}$ \\
Vacuum channel gap $C$ & $2.0 \mu \mathrm{m}$ \\
Pillar height $H$ & $1.0 \mu \mathrm{m}=0.50 \lambda_{\mathrm{p}}$ \\
Pillar width $A$ & $1.6 \mu \mathrm{m}=0.80 \lambda_{\mathrm{p}}$ \\
Misalignment level $\Delta$ & $1.4 \mu \mathrm{m}=0.70 \lambda_{\mathrm{p}}$ \\
\hline \hline
\end{tabular}

$E_{\mathrm{m}}=7.60 \mathrm{GV} / \mathrm{m}$ is still under the damage threshold, which yields an unloaded gradient $G=1.29 \mathrm{GV} / \mathrm{m}$ according to $G$ / $E_{\mathrm{m}}=0.170$ from the geometry optimization studies. In its comoving frame, the electrons experience a temporal electric field $E_{\mathrm{t}}=G_{\mathrm{p}} e^{-\left(\frac{z}{w_{\text {int }}}\right)^{2}}$ with a characteristic interaction length $w_{\text {int }}=\left(\frac{1}{w_{z}^{2}}+\frac{2 \ln 2}{(\beta c \tau)^{2}}\right)^{-0.5}=23 \mu \mathrm{m}$. Integration of this field $E_{\mathrm{t}}$ with a peak accelerating gradient of $G_{\mathrm{p}}=1.0 \mathrm{GV} / \mathrm{m}$ results in a maximum energy gain of $\Delta E_{\mathrm{m}}=40 \mathrm{keV}$, which can be used to calculate the accelerating gradient for subsequent simulations.

\section{Electron bunch parameters}

CLARA will be a normal conducting linear electron accelerator capable of accelerating electrons to $250 \mathrm{MeV}$ with a bunch charge of up to $250 \mathrm{pC}$. Table III shows the detailed CLARA bunch parameters reproduced from Ref. 22.

When CLARA works in an ultra-short pulse mode, ${ }^{22}$ a short electron bunch with a longitudinal RMS length of $9 \mu \mathrm{m}$ can be generated. When such a bunch is transmitted through the energy collimators, a bunch with a charge of $0.1 \mathrm{pC}$ and an energy spread of $0.05 \%$ is expected. Then, it can be focused by a permanent quadrupole magnet to give a transverse RMS radius of $10 \mu \mathrm{m}$, as presented in Table III before injecting into the optimum dual-grating structure. Here, the transverse size is much bigger than the vacuum channel gap of $1 \mu \mathrm{m}$, so only a small fraction of electrons traverse through the vacuum channel of the structure. Those electrons travelling through the quartz substrate and grating pillars suffer significant energy loss due to collisional straggling ${ }^{33}$ in the dielectric material, so they are excluded in our following analysis. All of our simulations only focus on those electrons modulated by the laser field in the vacuum channel for analysis in terms of emittance and energy spread, which can be

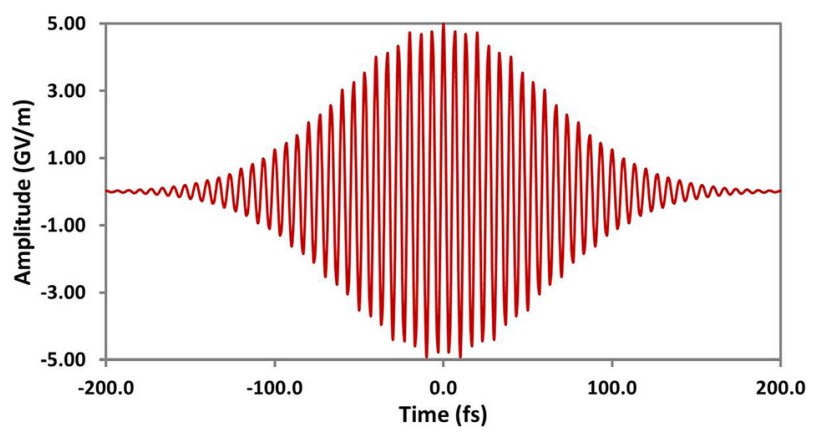

FIG. 4. The electric field envelope of the laser plane wave.
TABLE II. Parameters of the Gaussian laser plane wave used in the simulation.

\begin{tabular}{lc}
\hline \hline Laser characteristics \\
\hline Propagation direction \\
Wavelength $\lambda$ & $+\mathrm{y}$ \\
Peak laser field $E_{\mathrm{p}}$ & $2 \mu \mathrm{m}$ \\
FWHM duration $\tau$ & $5 \mathrm{GV} / \mathrm{m}$ \\
Waist radius $w_{\mathrm{z}}$ & $100 \mathrm{fs}$ \\
Frequency $f$ & $50 \mu \mathrm{m}$ \\
\hline \hline
\end{tabular}

TABLE III. CLARA bunch parameters used in our simulation

\begin{tabular}{lcc}
\hline \hline Bunch parameters & CLARA & Simulation \\
\hline Bunch energy $(\mathrm{MeV})$ & $50-250$ & 50 \\
Bunch charge $(\mathrm{pC})$ & $\leq 250$ & 0.1 \\
Bunch RMS length $(\mu \mathrm{m})$ & $9-300$ & 9 \\
Bunch RMS radius $(\mu \mathrm{m})$ & $10-100$ & 10 \\
Bunch density $\left(\mathrm{m}^{-3}\right)$ & $10^{18}-10^{22}$ & $4.4 \times 10^{19}$ \\
Normalized emittance $(\mathrm{mm} \cdot \mathrm{mrad})$ & $\leq 1$ & 0.2 \\
Energy spread & $0.01 \%-0.10 \%$ & $0.05 \%$ \\
\hline \hline
\end{tabular}

seen in Fig. 5. In addition, considering that electron energy of $50 \mathrm{MeV}$ is highly relativistic, a space charge is not taken into account in our simulations.

\section{The CLARA bunch travels through the optimum structure when laser is off}

In the first Particle-In-Cell (PIC) simulation, the CLARA electron bunch travels through the optimum structure without interacting with the laser. The excited wakefield is reflected back by dielectric gratings and interacts with the bunch itself; this may result in energy loss or deflection of those electrons in the bunch tails in terms of its final emittance and energy spread. 500000 macroparticles are used for our particle tracking simulations. It is found that about $4 \%$ of the $50 \mathrm{MeV}$ bunch is transmitted through the vacuum

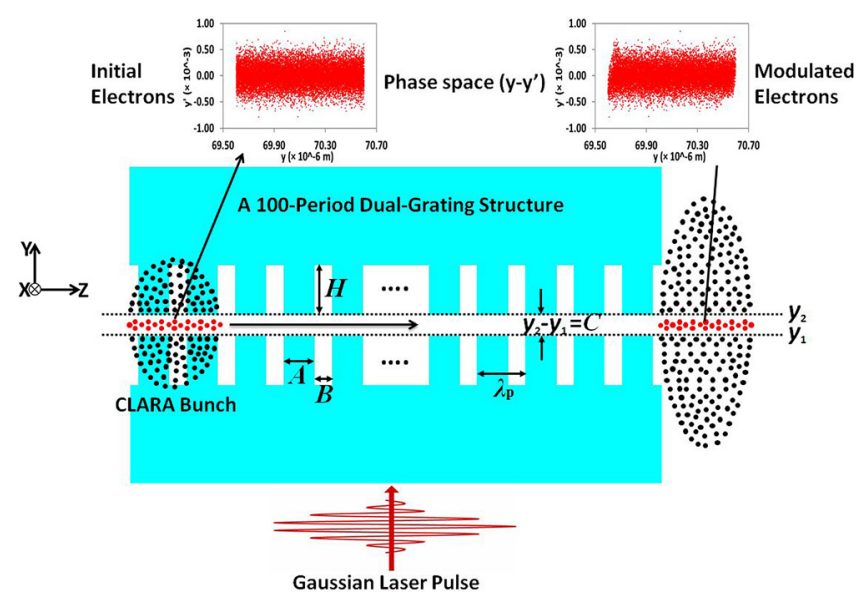

FIG. 5. Schematic of a CLARA electron bunch travelling through the optimum structure to interact with a Gaussian laser pulse, where the phase spaces for initial and modulated electrons are shown in subplots. 

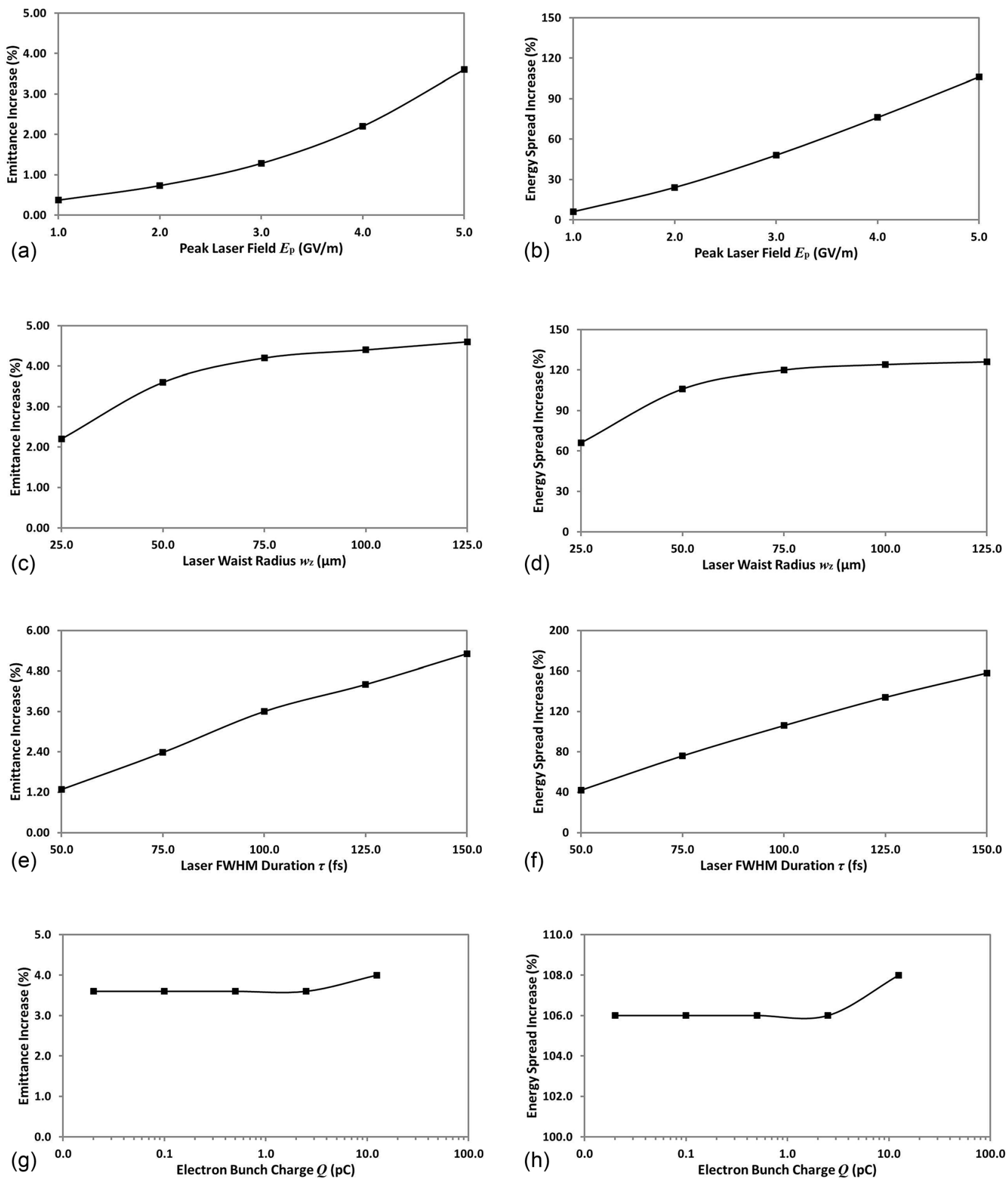

FIG. 6. Emittance ((a), (c), (e), and (g)) and energy spread ((b), (d), (f), and (h)) for modulated electrons as functions of a peak laser field $E_{\mathrm{p}}\left(w_{\mathrm{z}}=50 \mu \mathrm{m}\right.$, $\tau=100 \mathrm{fs}, Q=0.1 \mathrm{pC})$, a laser waist radius $w_{\mathrm{z}}\left(E_{\mathrm{p}}=5.0 \mathrm{GV} / \mathrm{m}, \tau=100 \mathrm{fs}, Q=0.1 \mathrm{pC}\right)$, a laser FWHM duration $\tau\left(E_{\mathrm{p}}=5.0 \mathrm{GV} / \mathrm{m}, w_{\mathrm{z}}=50 \mu \mathrm{m}, Q=0.1 \mathrm{pC}\right)$, and an electron bunch charge $Q\left(E_{\mathrm{p}}=5.0 \mathrm{GV} / \mathrm{m}, w_{\mathrm{z}}=50 \mu \mathrm{m}, \tau=100 \mathrm{fs}\right)$.

channel gap of $1.0 \mu \mathrm{m}$. When the bunch travels out of the optimum structure, the charge is $3.9 \mathrm{fC}$, the transverse RMS normalized emittance is $5.5 \mathrm{~nm}$, and the energy spread is $0.05 \%$ for those electrons between the $y_{1}$ and $y_{2}$ planes (see Fig. 5). The final bunch energy spectrum as shown in Fig. 6, which is calculated from the results of each individual particle trace, indicates that the longitudinal wakefield has a weak force to the bunch itself after comparing with initial spectrum. This could also be used to compare with that of the following laser-on case.

\section{E. The CLARA bunch travels through the optimum structure when the laser is on}

In the second simulation scenario, the CLARA electron bunch is injected into the optimum structure to interact with 
the laser as summarized in Table II. From particle tracking simulations, it is found that the transverse RMS normalized emittance is $5.7 \mathrm{~nm}$ and the RMS energy spread is $0.103 \%$ for those modulated electrons when the bunch travels out of the structure (see Fig. 5), corresponding to increases of $3.6 \%$ for emittance and $106 \%$ for energy spread compared to those of the laser-off case. With an RMS bunch length of $9 \mu \mathrm{m}$, the electrons are able to sample all phases of the laser field in the channel gap, causing some electrons to gain energy from acceleration, while others are decelerated, which generates a big energy spread. The minor emittance difference may be explained by a weak deflecting force excited by the laser plane wave. However, it also indicates that the laser field inside the structure does not have an obvious effect on the final bunch transverse emittance at such short interaction length $\left(w_{\text {int }}=23 \mu \mathrm{m}\right)$.

Later, we study some factors influencing the final beam quality in terms of emittance and energy spread in detail. Figures 6(a) and 6(b) illustrate that the emittance and energy spread rise with a larger peak laser field, which induces a stronger accelerating and deflecting field for electrons to experience. For a peak laser field of $1 \mathrm{GV} / \mathrm{m}$, small increases of $0.4 \%$ for emittance and $6 \%$ for energy spread are expected, but at the cost of a reduced accelerating gradient. With a bigger laser waist radius and FWHM duration, the emittance and energy spread increase as shown in Figs. 6(c)-6(f). This is expected since the interaction length $w_{\text {int }}$ is related to laser waist radius and FWHM duration. Figures $6(\mathrm{~g})$ and $6(\mathrm{~h})$ show that the emittance and energy spread remain constant when the bunch charge $Q$ is between $0.02 \mathrm{pC}$ and $2.5 \mathrm{pC}$, and increase when $Q>2.5 \mathrm{pC}$, which corresponds to a bunch density of $1.1 \times 10^{21} / \mathrm{m}^{3}$. This means that a bunch density higher than $1.1 \times 10^{21} / \mathrm{m}^{3}$ can excite a stronger longitudinal and transverse wakefield, which results in larger emittance and energy spread. Based on these analyses, a high peak laser field with long waist radius and FWHM duration is preferred to achieve a considerable energy gain in a long-range acceleration; however, the resulting emittance increase could be an issue. A low bunch density of $<1.1 \times 10^{21} / \mathrm{m}^{3}$ is desired to load into such a dualgrating structure to interact with laser pulses, in which a small emittance increase of $\sim 3.6 \%$ can be achieved. In addition, the deflecting force can be compensated for by symmetric illumination using two laser beams from opposite sides.

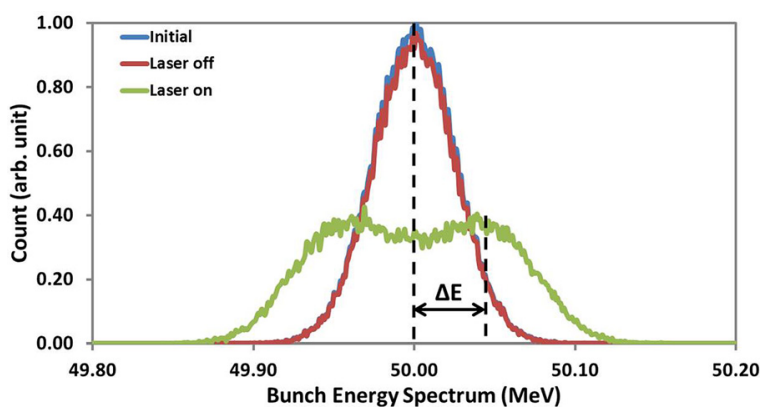

FIG. 7. Bunch energy spectrum for initial electrons and modulated electrons with the laser off and on. Electrons travelling through the quartz substrate and grating pillars are not shown in this figure.
The CLARA electron bunch can be partitioned into a series of short slices of length $\Delta t \ll \lambda_{0} / c$. After interacting with the sinusoidal electric field in the channel gap of the structure, each slice of the electron bunch experiences a net energy shift described by

$$
g(\Delta t, \Delta E)=\Delta E \cos \left(\frac{2 \pi c}{\lambda_{0}} \Delta t\right)
$$

where $\Delta E$ is the maximum energy gain for the electrons. For a bunch with a Gaussian distributed energy spectrum, when all contributions from each slice are superimposed, it is easily predicted that the energy spectrum will be changed to a double-peaked profile, ${ }^{26}$ as shown in Fig. 7. It demonstrates that the maximum energy gain is $\Delta E=45 \pm 6 \mathrm{keV}$, corresponding to a maximum accelerating gradient of $G=1.13$ $\pm 0.15 \mathrm{GV} / \mathrm{m}$. Given that the maximum electric field $E_{\mathrm{m}}$ $=7.60 \mathrm{GV} / \mathrm{m}$ in the structure observed from VSim, the accelerating factor $A F=G / E_{\mathrm{m}}=0.149 \pm 0.020$ can be compared with the unloaded $A F=0.170$ from the finite-difference timedomain simulation for dual-grating structures.

\section{CONCLUSION}

This paper presents detailed beam quality studies for an optimized 100-period dual-grating structure in terms of the emittance, energy spread, and loaded accelerating gradient. In order to get the optimum geometry, we have carried out studies to maximize the accelerating factor for quartz dual-grating structures. For dual-grating structures with a vacuum channel gap of half laser wavelength illuminated by a single laser beam, a maximum accelerating factor $A F=0.170$ can be obtained, corresponding to a maximum unloaded gradient of $G=1.53 \mathrm{GV} / \mathrm{m}$. Using VSim, a realistic CLARA electron bunch with a longitudinal bunch length of $9 \mu \mathrm{m}$ and a transverse RMS size of $10 \mu \mathrm{m}$ is loaded into the optimum dual-grating structure to interact with a realistic laser pulse. Our numerical simulations only focus on electrons travelling through the vacuum channel to interact with laser field. In this case, when the modulated electrons travel out of the structure, the transverse normalized emittance increases by $3.60 \%$ compared to that of laser-off case, the energy spread changes from $0.05 \%$ to $0.103 \%$, and an accelerating gradient up to $1.13 \pm 0.15 \mathrm{GV} / \mathrm{m}$ could be expected from the particle tracking simulations. In addition, we also analyze the effect of laser parameters and electron density on the beam quality in detail. When electrons interact with a higher peak laser field with longer waist radius and FWHM duration, it can lead to the increase of emittance and energy spread. However, a low electron bunch density of $<1.1$ $\times 10^{21} / \mathrm{m}^{3}$ is desired to generate a small emittance increase of $\sim 3.6 \%$.

These simulation results not only theoretically demonstrate the capabilities of a dual-grating DLA with good beam quality but also numerically predict the realistic DLA experiment results in terms of emittance, energy spread, and loaded gradient. However, the energy gain in our simulations is limited by the laser-electron interaction length. A pulse-front- 
tilt operation for a laser beam will be considered as part of future studies to extend the interaction length, thereby resulting in a greater energy gain for a DLA.

Dual-grating structures have been fabricated at the Laboratory for Micro and Nanotechnology, PSI (Paul Scherrer Institute). They will be tested with beam in 2017 and benchmarked against results from simulations to further optimize both the structure and the acceleration process overall.

\section{ACKNOWLEDGMENTS}

We would like to thank Dr. Lee Devlin for carefully proof reading the original manuscript and Dr. Rasmus Ischebeck, Dr. Micha Dehler, Dr. Eugenio Ferrari, and Dr. Nicole Hiller for many useful discussions. This work was supported by the EU under Grant Agreement No. 289191, the STFC Cockcroft Institute core Grant No. ST/G008248/1.

${ }^{1}$ J. Norem, V. Wu, A. Moretti, M. Popovic, Z. Qian, L. Ducas, Y. Torun, and N. Solomey, Phys. Rev. Spec. Top. Accel. Beams 6, 072001 (2003).

${ }^{2}$ A. Moretti, Z. Qian, J. Norem, Y. Torun, D. Li, and M. Zisman, Phys. Rev. Spec. Top. Accel. Beams 8, 072001 (2005).

${ }^{3}$ M. R. Jana, M. Chung, M. Leonova, A. Moretti, A. Tollestrup, and K. Yonehara, in Proceedings of PAC2013, Pasadena, CA, USA, p. WEPMA12.

${ }^{4}$ T. Plettner, P. P. Lu, and R. L. Byer, Phys. Rev. Spec. Top. Accel. Beams 9, 111301 (2006).

${ }^{5}$ A. Aimidula, M. A. Bake, F. Wan, B. S. Xie, C. P. Welsch, G. Xia, O. Mete, M. Uesaka, Y. Matsumura, M. Yoshida, and K. Koyama, Phys. Plasmas 21, 023110 (2014).

${ }^{6}$ A. Aimidula, C. P. Welsch, G. Xia, K. Koyama, M. Uesaka, M. Yoshida, O. Mete, and Y. Matsumura, Nucl. Instrum. Methods Phys. Res., Sect. A 740, 108 (2014).

${ }^{7}$ C. M. Chang and O. Solgaard, Appl. Phys. Lett. 104, 184102 (2014).

${ }^{8}$ X. E. Lin, Phys. Rev. Spec. Top. Accel. Beams 4, 051301 (2001).

${ }^{9}$ V. Reboud, J. Romero-Vivas, P. Lovera, N. Kehagias, T. Kehoe, G. Redmond, and C. M. S. Torres, "Lasing in nanoimprinted twodimensional photonic crystal band-edge lasers," Appl. Phys. Lett. 102, 073101 (2013)

${ }^{10}$ B. M. Cowan, Phys. Rev. Spec. Top. Accel. Beams 6, 101301 (2003).

${ }^{11}$ Z. Wu, R. J. England, C. K. Ng, B. Cowan, C. McGuinness, C. Lee, M. Qi, and S. Tantawi, Phys. Rev. Spec. Top. Accel. Beams 17, 081301 (2014).

${ }^{12}$ T. Plettner and R. L. Byer, Phys. Rev. Spec. Top. Accel. Beams 11, 030704 (2008)

${ }^{13}$ K. Soong and R. L. Byer, Opt. Lett. 37, 975-977 (2012).

${ }^{14}$ T. Plettner, R. L. Byer, C. McGuinness, and P. Hommelhoff, Phys. Rev. Spec. Top. Accel. Beams 12, 101302 (2009).
${ }^{15}$ E. A. Peralta, K. Soong, R. J. England, E. R. Colby, Z. Wu, B. Montazeri, C. McGuinness, J. McNeur, K. J. Leedle, D. Walz, E. B. Sozer, B. Cowan, B. Schwartz, G. Travish, and R. L. Byer, Nature 503, 91 (2013).

${ }^{16}$ K. P. Wootton, Z. Wu, B. M. Cowan, A. Hanuka, I. V. Makasyuk, E. A. Peralta, K. Soong, R. L. Byer, and R. J. England, Opt. Lett. 41, 2696-2699 (2016).

${ }^{17}$ J. Breuer and P. Hommelhoff, Phys. Rev. Lett. 111, 134803 (2013).

${ }^{18}$ K. J. Leedle, R. F. Pease, R. L. Byer, and J. S. Harris, Optica 2, 158 (2015).

${ }^{19}$ K. J. Leedle, A. Ceballos, H. Deng, O. Solgaard, R. F. Pease, R. L. Byer, and J. S. Harris, Opt. Lett. 40, 4344-4347 (2015).

${ }^{20}$ Y. Wei, C. P. Welsch, G. Xia, O. Mete, K. Hanahoe, and J. D. A. Smith, in Proceedings of the IPAC2015, Richmond, VA, USA, p. WEPWA051.

${ }^{21}$ B. Montazeri Najafabadi, R. L. Byer, C. K. Ng, R. J. England, E. A. Peralta, K. Soong, R. Noble, and Z. Wu, AIP Conf. Proc. 1507, 476 (2012).

${ }^{22}$ J. A. Clarke, D. Angal-Kalinin, N. Bliss, R. Buckley, S. Buckley, R. Cash, P. Corlett, L. Cowie, G. Cox, G. P. Diakun, D. J. Dunning, B. D. Fell, A. Gallagher, P. Goudket, A. R. Goulden, D. M. P. Holland, S. P. Jamison, J. K. Jones, A. S. Kalinin, W. Liggins, L. Ma, K. B. Marinov, B. Martlew, P. A. McIntosh, J. W. McKenzie, K. J. Middleman, B. L. Militsyn, A. J. Moss, B. D. Muratori, M. D. Roper, R. Santer, Y. Saveliev, E. Snedden, R. J. Smith, S. L. Smith, M. Surman, T. Thakker, N. R. Thompson, R. Valizadeh, A. E. Wheelhouse, P. H. Williams, R. Bartolini, I. Martin, R. Barlow, A. Kolano, G. Burt, S. Chattopadhyay, D. Newton, A. Wolski, R. B. Appleby, H. L. Owen, M. Serluca, G. Xia, S. Boogert, A. Lyapin, L. Campbell, B. W. J. McNeil, and V. V. Paramonov, J. Instrum. 9, T05001 (2014).

${ }^{23}$ R. B. Palmer, Part. Accel. 11, 81-90 (1980); available at http://cds.cern.ch/ record/1107986/files/p81.pdf.

${ }^{24}$ D. M. Pai and K. A. Awada, Opt. Soc. Am. A 8, 755-762 (1991).

${ }^{25}$ J. Breuer, J. McNeur, and P. Hommelhoff, J. Phys. B: At. Mol. Opt. Phys. 47, 234004 (2014).

${ }^{26}$ E. A. Peralta, "Accelerator on a chip: Design, fabrication, and demonstration of grating-based dielectric microstructures for laser-driven acceleration of electrons," Ph.D. thesis (Stanford University, 2015).

${ }^{27}$ See https://www.txcorp.com/vsim for VSim.

${ }^{28}$ T. Plettner, R. L. Byer, and B. Montazeri, J. Mod. Opt. 58, 1518 (2011).

${ }^{29}$ R. Kitamura, L. Pilon, and M. Jonasz, Appl. Opt. 46, 8118 (2007).

${ }^{30}$ R. J. England, R. J. Noble, K. Bane, D. H. Dowell, C.-K. Ng, J. E. Spencer, S. Tantawi, Z. Wu, R. L. Byer, E. Peralta, K. Soong, C. Chang, B. Montazeri, S. J. Wolf, B. Cowan, J. Dawson, W. Gai, P. Hommelhoff, Y. Huang, C. Jing, C. McGuinness, R. B. Palmer, B. Naranjo, J. Rosenzweig, G. Travish, A. Mizrahi, L. Schachter, C. Sears, G. R. Werner, and R. B. Yoder, Rev. Mod. Phys. 86, 1337 (2014).

${ }^{31}$ M. Lenzner, J. Krüger, S. Sartania, Z. Cheng, Ch. Spielmann, G. Mourou, W. Kautek, and F. Krausz, Phys. Rev. Lett. 80, 4076 (1998).

${ }^{32}$ K. Soong, R. L. Byer, C. McGuinness, E. Peralta, and E. Colby, in Proceedings of 2011 Particle Accelerator Conference (PAC2011) (IEEE, New York, USA, 2011), p. MOP095.

${ }^{33}$ C. Warner III and F. Rohrlich, Phys. Rev. 93(3), 406 (1954). 